\title{
EFFECT OF DIFFUSION ON BUNCHED BEAM ECHO
}

\author{
G. V. Stupakov and A. W. Chao \\ Stanford Linear Accelerator Center, Stanford University, Stanford, CA 94309
}

\begin{abstract}
When a beam receives a dipole kick, its centroid signal decoheres due to the betatron tune spread in the beam. Long after the signal has decohered, however, a followup quadrupole kick to the beam brings a pronounced echo back to the centroid signal. This echo effect has been analyzed for the case of a bunched beam in Ref. [1]. In this work, the perturbation calculation of Ref. [1] is extended to include a diffusion in betatron amplitude. The effect of diffusion on the magnitude of the echo is then parameterized and studied.
\end{abstract}

\section{INTRODUCTION}

The echo effect has been known in plasma physics for many years (see, e.g., [2]). Relatively recently a concept of echo has been introduced into accelerator physics $[1,3]$. For a bunched beam, the echo in a circular accelerator can be observed when the beam is kicked off-center at time $t=0$ causing its centroid to undergo betatron oscillations. After these oscillations completely damped out due to beam decoherence, the beam is excited by a quadrupole kick at time $t=\tau$. This kick does not produce any visible beam centroid displacement at that time, but it turns out that close to time $t=2 \tau$ the beam centroid undergoes transient betatron oscillations with an amplitude that is a fraction of the initial beam offset. The echo can also be observed in the longitudinal direction [4] in which case RF phase shift and RF amplitude jump play a role of the dipole and quadrupole kicks, respectively.

Experimentally, longitudinal echo has been observed in the anti-proton accumulator ring at FNAL [5] and in the CERN SPS [6] for coasting beams. Those experiments demonstrated that echo can be effectively used for measuring an extremely weak diffusion inside the beam.

For a longitudinal echo in a coasting beam, the theory of echo effect taking into account the diffusion has been developed in Refs. [5, 7]. In the present paper we extend the theory of transverse echo to include the diffusion effects in a bunched beam.

\section{THEORY}

Following approach of Ref. [1] we will use the action - angle variables, $J$ and $\phi$, for description of transverse dynamics of a bunched beam. With diffusion, we need to solve the Fokker-Planck equation

$$
\frac{\partial \psi}{\partial t}+\omega_{\beta}(J) \frac{\partial \psi}{\partial \phi}=\frac{\partial}{\partial J}\left(D(J) J \frac{\partial \psi}{\partial J}\right),
$$

where $\psi(J, \phi)$ is the beam distribution function, $\omega_{\beta}(J)$ is the betatron frequency as a function of the amplitude of the betatron oscillations, and $D(J)$ is the diffusion coefficient.

We will solve Eq. (1) in the limit of weak diffusion. Specifically, we assume that the diffusion has a small effect on a time scale during which the beam decoheres. The decoherence time, $\tau_{\text {decoh }}$, can be estimated as $\tau_{\text {decoh }} \sim$ $1 / \omega_{\beta}^{\prime} J$, and the diffusion time, $\tau_{d i f}$, is roughly equal to $\tau_{\text {dif }} \sim J / D$. Requiring $\tau_{\text {dif }} \gg \tau_{\text {decoh }}$ we get

$$
D \ll \omega_{\beta}^{\prime} J^{2} .
$$

In the limit of very strong diffusion (typical for electron accelerators where diffusion is caused by quantum fluctuations of the synchrotron radiation), when the inequality opposite to (2) holds, the diffusion completely suppresses the echo effect.

For $t<0$, we assume an initial distribution function,

$$
\psi=\psi_{0}(J)
$$

At time $t=0$, the beam receives a small dipole kick such that the amplitude of the dipole oscillation is much smaller than the beam size. Immediately after the kick we have from Ref. [1] that the perturbation $\psi_{1}$ of the distribution function is

$$
\psi_{1}(J, u)=\epsilon \sqrt{2 J} \psi_{0}^{\prime}(J) \sin u,
$$

where $\epsilon$ gives the strength of the kick, $\epsilon \ll 1$, and $u=$ $\phi-\omega_{\beta}(J) t$.

In the period $0<t<\tau$, changing variable from $(J, \phi, t)$ to $(J, u, t)$ in Eq. (1) gives for $\psi_{1}$

$$
\begin{aligned}
\frac{\partial \psi_{1}}{\partial t} & =\left(\frac{\partial}{\partial J}-\omega_{\beta}^{\prime}(J) t \frac{\partial}{\partial u}\right) \\
& \times\left[D(J) J\left(\frac{\partial \psi_{1}}{\partial J}-\omega_{\beta}^{\prime}(J) t \frac{\partial \psi_{1}}{\partial u}\right)\right] .
\end{aligned}
$$

When $\left|\omega_{\beta}^{\prime}\right| t J \gg 1$, Eq. (5) becomes

$$
\frac{\partial \psi_{1}}{\partial t} \approx\left[\omega_{\beta}^{\prime}(J) t\right]^{2} D(J) J \frac{\partial^{2} \psi_{1}}{\partial u^{2}} .
$$

With initial condition (4), the solution of (6) for $0<t<\tau$ is

$$
\psi_{1}(J, u, t)=\epsilon \sqrt{2 J} \psi_{0}^{\prime}(J) e^{-\frac{1}{3} D(J) J\left(\omega_{\beta}^{\prime}(J)\right)^{2} t^{3}} \sin u .
$$

At $t=\tau$, the beam receives a quadrupole kick, after which the perturbation $\psi_{2}$ is (see Ref. [1])

$$
\begin{aligned}
& \psi_{2}(J, u)=\sqrt{2} q \epsilon \omega_{\beta}^{\prime}(J) \tau J^{3 / 2} \psi_{0}^{\prime}(J) \\
& \times \exp \left[-\frac{1}{3} D(J) J\left(\omega_{\beta}^{\prime}(J)\right)^{2} \tau^{3}\right] \sin \left(2 u+2 \omega_{\beta}(J) \tau\right) \\
& \times \cos u
\end{aligned}
$$


where $q$ gives the strength of the quadrupole kick. Using a trigonometric identity, we can expand the product $\sin (2 u+$ $\left.2 \omega_{\beta}(J) \tau\right) \cos u$ into a sum of the first $\left(\frac{1}{2} \sin \left(u+2 \omega_{\beta}(J) \tau\right)\right)$ and the third $\left(\frac{1}{2} \sin \left(3 u+2 \omega_{\beta}(J) \tau\right)\right)$ harmonics with respect to the variable $u$. From these two terms only the first harmonic is responsible for the dipole echo; the second term gives rise to a sextupole echo which we omit in what follows.

In the period $t>\tau$, we make a change of variable from $(J, \phi, t)$ to $\left(J, u_{1}, t\right)$ where $u_{1}=\phi-\omega_{\beta}(J)(t-2 \tau)$ in Eq. (1) to obtain for $\psi_{2}$

$$
\begin{aligned}
& \frac{\partial \psi_{2}}{\partial t}=\left(\frac{\partial}{\partial J}-\omega_{\beta}^{\prime}(J)(t-2 \tau) \frac{\partial}{\partial u_{1}}\right) \\
& \times\left[D(J) J\left(\frac{\partial \psi_{2}}{\partial J}-\omega_{\beta}^{\prime}(J)(t-2 \tau) \frac{\partial \psi_{2}}{\partial u_{1}}\right)\right] .
\end{aligned}
$$

When $\left|\omega_{\beta}^{\prime}(t-2 \tau)\right| J \gg 1$, we have

$$
\frac{\partial \psi_{2}}{\partial t} \approx\left[\omega_{\beta}^{\prime}(J)(t-2 \tau)\right]^{2} D(J) J \frac{\partial^{2} \psi_{2}}{\partial u_{1}^{2}} .
$$

The solution with the initial condition (8) (keeping only the term $\left.\propto \sin \left(u+2 \omega_{\beta}(J) \tau\right)\right)$ is

$$
\begin{aligned}
\psi & =\frac{1}{\sqrt{2}} q \epsilon \omega_{\beta}^{\prime}(J) \tau J^{3 / 2} \psi_{0}^{\prime}(J) \\
& \times \sin \left(\phi-\omega_{\beta}(J) t+2 \omega_{\beta}(J) \tau\right) \\
& \times \exp \left[-\frac{1}{3} D(J) J\left(\omega_{\beta}^{\prime}(J)\right)^{2}\left((t-2 \tau)^{3}+2 \tau^{3}\right)\right] .
\end{aligned}
$$

Now, we have to note that although Eq. (10) is not valid in the vicinity of $t=2 \tau$ due to the approximation made in Eq. (10), the solution (11) turns out to be approximately valid for all times. The reason for that is that duration of the period when $|(t-2 \tau)|<1 / \omega_{\beta}^{\prime} J$ is of the order of the decoherence time, and according to our assumption (2) the diffusion is negligible during that period. Formally, the right hand side of Eq. (10) becomes small when $|(t-2 \tau)|$ approaches zero, and we can neglect it putting $\partial \psi_{2} / \partial t \approx 0$ during that period, from which is follows that $\psi_{2} \approx$ const. On the other hand, we find that solution (11) indeed does not change noticeably during this interval.

The centroid offset of the bunch is given by

$$
\bar{\eta}(t)=\int_{0}^{\infty} d J \sqrt{2 J} \int_{0}^{2 \pi} d \phi \cos \phi \psi(J, \phi, t) .
$$

Substituting (11) into (12) gives for the echo signal

$$
\begin{aligned}
\bar{\eta}^{\text {echo }}(t) & =-\pi q \epsilon \tau \int_{0}^{\infty} d J \omega_{\beta}^{\prime}(J) J^{2} \psi_{0}^{\prime}(J) \\
& \times \sin \left[\omega_{\beta}(J)(t-2 \tau)\right] \\
& \times e^{-\frac{1}{3} D(J) J\left(\omega_{\beta}^{\prime}(J)\right)^{2}\left(2 \tau^{3}+(t-2 \tau)^{3}\right)} .
\end{aligned}
$$

Eq. (13) can be cast into a complex form by substituting $\sin \left[\omega_{\beta}(J)(t-2 \tau)\right]$ by $\exp \left[i \omega_{\beta}(J)(t-2 \tau)\right]$ that defines a complex echo signal of which the real echo is its imaginary part,

$$
\begin{aligned}
& \bar{\eta}^{\text {echo }}(t)=-\pi q \epsilon \tau \int_{0}^{\infty} d J \omega_{\beta}^{\prime}(J) J^{2} \psi_{0}^{\prime}(J) \\
& \times e^{i \omega_{\beta}(J)(t-2 \tau)} e^{-\frac{1}{3} D(J) J\left(\omega_{\beta}^{\prime}(J)\right)^{2}\left(2 \tau^{3}+(t-2 \tau)^{3}\right)} .
\end{aligned}
$$

Note that the echo appears when the argument of the first exponent in the integrand of Eq. (14) approaches zero, $t \approx$ $2 \tau$. Since we assume that diffusion is small (see Eq. (2)), the second exponent is a slow function of time, and we can put $t=2 \tau$ in it,

$$
\begin{aligned}
\bar{\eta}^{\mathrm{echo}}(t) & \approx-\pi q \epsilon \tau \int_{0}^{\infty} d J \omega_{\beta}^{\prime}(J) J^{2} \psi_{0}^{\prime}(J) \\
& \times e^{i \omega_{\beta}(J)(t-2 \tau)} e^{-\frac{2}{3} D(J) J\left(\omega_{\beta}^{\prime}(J)\right)^{2} \tau^{3}} .
\end{aligned}
$$

Assuming that $\psi_{0}^{\prime}(J)$ is a monotonous function of its argument and using Schwarz's inequality, one can conclude that the maximum amplitude of echo occurs at $t=2 \tau$, and is equal to the following expression

$$
\begin{aligned}
& \left|\bar{\eta}^{\text {max echo ampl }}\right|=\left|\bar{\eta}^{\text {echo }}(2 \tau)\right| \\
= & -\pi q \epsilon \tau \int_{0}^{\infty} d J \omega_{\beta}^{\prime}(J) J^{2} \psi_{0}^{\prime}(J) e^{-\frac{2}{3} D(J) J\left(\omega_{\beta}^{\prime}(J)\right)^{2} \tau^{3}} .
\end{aligned}
$$

Eq. (15) is our main result. Given $\omega_{\beta}(J), \psi_{0}(J)$ and $D(J)$, it gives the echo response as a function of time. In the special case when

$$
\begin{aligned}
& \omega_{\beta}(J)=\omega_{0}+\omega^{\prime} J, \\
& \psi_{0}(J)=\frac{1}{2 \pi J_{0}} e^{-J / J_{0}},
\end{aligned}
$$

and assuming a constant diffusion coefficient, $D(J)=D_{0}$, the integration in (15) can be performed explicitly. The result is

$$
\bar{\eta}^{\mathrm{echo}}(t)=\frac{q \epsilon \omega^{\prime} \tau J_{0}}{(\beta-i \xi)^{3}} e^{i \Phi},
$$

where

$$
\begin{aligned}
\xi & =\omega^{\prime} J_{0}(t-2 \tau), \\
\beta & =1+\frac{2}{3} D_{0}\left(\omega^{\prime}\right)^{2} J_{0} \tau^{3}, \\
\Phi & =\omega_{0}(t-2 \tau) .
\end{aligned}
$$

When $D_{0}=0$, this reduces to Ref. [1].

One may calculate the amplitude of the dipole echo,

$$
\left|\bar{\eta}^{\text {echo ampl }}(t)\right|=\frac{q \epsilon \omega^{\prime} J_{0} \tau}{\left(\beta^{2}+\xi^{2}\right)^{3 / 2}} .
$$

The plot of this function is shown in Fig. 1. As mentioned above, the maximum value of the echo is achieved at time $t=2 \tau$, and the amplitude of the echo rolls off as $1 /|t-2 \tau|^{3}$ for large values of $|t-2 \tau|$. For small values of $\tau$ such that $\beta \approx 1$, the width of the echo pulse is of the order of the decoherence time $\tau_{\text {decoh }}$; it increases for larger $\tau$. 


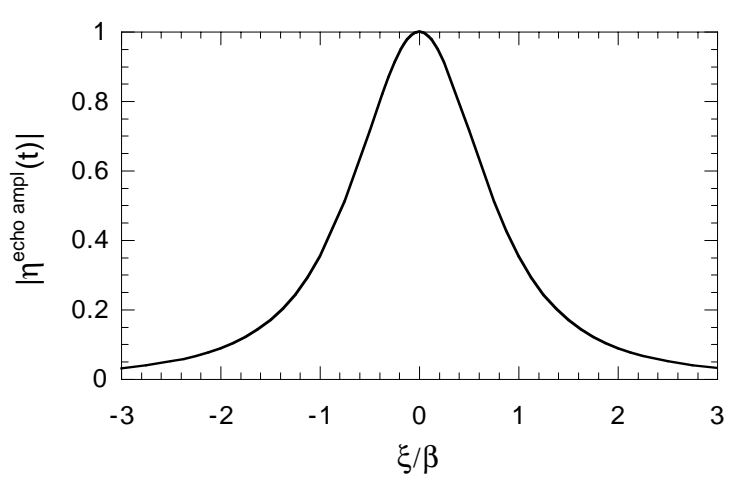

Figure 1: Echo signal as a function of the variable $\xi$ normalized to its maximum value.

Fig. 2 shows the maximum value of the echo amplitude as a function of the time $\tau$ between the dipole and quadrupole kicks. The maximum value of the echo amplitude is achieved at $\tau=\tau_{\max }=0.91\left(D_{0}\left(\omega_{0}^{\prime}\right)^{2} J_{0}\right)^{-1 / 3}$. In terms of decoherence and diffusion times, the maximum is achieved at $\tau_{\max } \sim\left(\tau_{\text {dif }} \tau_{\text {decoh }}^{2}\right)^{1 / 3}$.

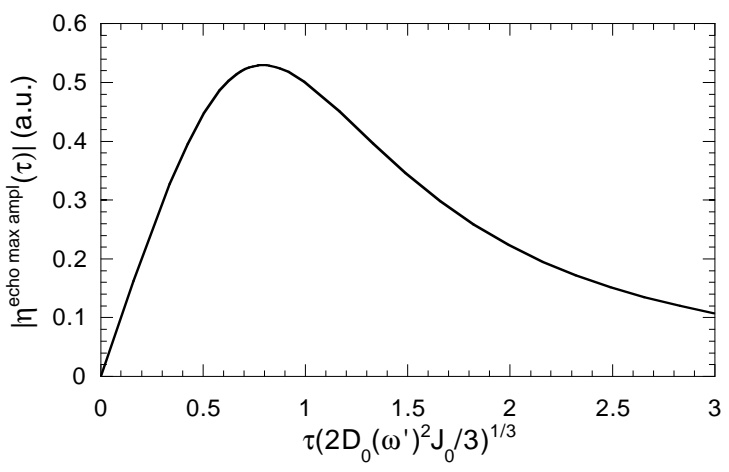

Figure 2: The maximum amplitude value of the echo as a function of the kick time $\tau$.

Experimentally, both quantities $\omega^{\prime}$ and $D_{0}$ can be found from the echo measurements. The full width at half maximum of the echo signal in the limit of small $\tau$ (when $\beta \approx 1$ ) is equal to $1.53 J_{0} \omega^{\prime}$. If beam emittance $J_{0}$ is known, this allows to determine $\omega^{\prime}$. After that $D_{0}$ can be found as $D_{0}=0.75 \tau_{\max }^{3} /\left(\omega_{0}^{\prime}\right)^{2} J_{0}$.

More complex dependences of the diffusion coefficient $D(J)$ can also be treated. We consider here the maximum echo amplitude for the case when $D(J)$ can be approximated by a power function,

$$
D(J)=D_{n} J^{n},
$$

and still assuming Eq. (17). From Eq. (16) we have

$$
\left|\bar{\eta}^{\text {max echo ampl }}\right|=\frac{1}{2} \pi q \epsilon \omega^{\prime} J_{0} \tau_{n} F_{n}\left(\frac{\tau}{\tau_{n}}\right),
$$

where

$$
\tau_{n}=\left(\frac{2}{3}\left(\omega^{\prime}\right)^{2} D_{n} J_{0}^{n+1}\right)^{-1 / 3}
$$

and

$$
F_{n}(\zeta)=\zeta \int_{0}^{\infty} d x x^{2} \exp \left(-x-x^{n+1} \zeta^{3}\right) .
$$

Fig. 3 shows the plots of the functions $F_{n}$ for $\mathrm{n}=1,2$ and 3 .

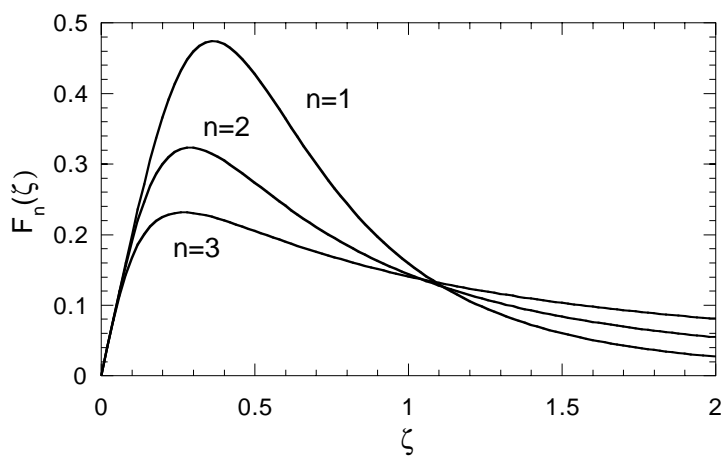

Figure 3: Plot of functions $F_{n}$ for $\mathrm{n}=1,2$ and 3 .

\section{CONCLUSION}

We extended the perturbation theory of the transverse echo effect in a bunched beam to include the diffusion. Without diffusion, the perturbation theory predicts a linear growth of the echo signal with the delay time $\tau$ (which is only true if the the echo signal is small; see nonperturbative approach in Ref. [3]). With diffusion, we find that the echo signal reaches maximum at $\tau_{\max } \sim\left(\tau_{\text {dif }} \tau_{\text {decoh }}^{2}\right)^{1 / 3}$, and vanishes when $\tau \gg \tau_{\max }$. The exact value of $\tau_{\max }$ depends on the functional form of the diffusion coefficient and is calculated above for several simple power dependences. Experimentally, transverse echo gives a possibility not only to measure the diffusion coefficient within the bunch, but also to distinguish between different dependences $D(J)$.

\section{ACKNOWLEDGMENT}

This work was supported by Department of Energy contract DE-AC03-76SF00515.

\section{REFERENCES}

[1] G. Stupakov. "Echo Effect in Hadron Colliders", SSCL Report 579 (1992).

[2] T.M. Malmberg, Phys. Rev. Letters, 19, 219 (1967).

[3] G. Stupakov and S. Kauffmann, "Echo Effect in Accelerators", SSCL Report 587 (1992).

[4] N. Mahale et al. SSCL Report No. SSCL-N-817, 1993.

[5] L.K. Spenzouris, J.-F. Ostigy, P.L. Colestock, Phys. Rev. Letters 76, 620 (1996).

[6] O. Bruning et al. "Longitudinal Beam Echo in the CERN SPS", EPAC 96.

[7] O. Bruning. "On the Possibility of Measuring Longitudinal Echos in the SPS", CERN SL/95-83 (AP), 1995. 